Check for updates

Cite this: RSC Adv., 2017, 7, 26082

Received 1st March 2017

Accepted 9th May 2017

DOI: $10.1039 / \mathrm{c} 7 \mathrm{ra02529g}$

rsc.li/rsc-advances

\title{
Flame retardant epoxy resin based on organic titanate and polyhedral oligomeric silsesquioxane- containing additives with synergistic effects $\dagger$
}

\author{
Birong Zeng, (D)* Yongzhou Liu, Li Yang, Wei Zheng, Ting Chen, Guorong Chen, \\ Yiting Xu, Conghui Yuan (D) and Lizong Dai (D)
}

\begin{abstract}
In order to develop a new epoxy resin (EP) possessing good thermal and mechanical properties as well as flame retardancy performance, the compound POSS-bisDOPO consisting of one polyhedral oligomeric silsesquioxane (POSS) and two unit of 9,10-dihydro-9-oxa-10-phosphaphenanthrene-10-oxide (DOPO) was synthesized and used as co-additive with tetrabutyl titanate to construct a phosphorous-silicontitanium synergy system. Compared to pure EP, the char yields of the EP composites were increased. The LOI value and UL-94 ratings of the EP composite with a loading of 5\% POSS-bisDOPO reached $31.0 \%$ and $\mathrm{V}-0$ grade, respectively. The PHRR and THR reduced to $28.4 \mathrm{~W} \mathrm{~g}^{-1}$ and $1.7 \mathrm{~kJ} \mathrm{~g}^{-1}$. SEM results indicated that the char of the EP composites had an alveolate inner layer and continual compact outer layer, which was conducive to form effectively physical barrier to hinder the penetrating of oxygen and heat. DMA and three-point bending test results showed the mechanical property of the EP composites had no decline.
\end{abstract}

\section{Introduction}

As a typical thermosetting material, epoxy resin has widespread applications in surface coatings, electronic encapsulating, adhesives and advanced composites matrices due to their excellent solvent resistance, low moisture absorption, strong adherence to many substrates, and outstanding electrical and mechanical properties. ${ }^{1}$ Although epoxy resin has many excellent performance, its application is still limited by high flammability. Like other resins, the flame resistance of epoxy resins can be enhanced by flame retardants. Halogenated compounds were widely used to improve the flame resistance and showed high efficiency. However, the use of traditional halogenated flame retardant will lead a series of environment concerns as they can produce poisonous and corrosive smoke and release halogenated dibenzo-dioxins and dibenzo-furans. ${ }^{2}$ Therefore, it is necessary to exploit halogen-free flame retardants to improve the flame resistance of epoxy resins.

Phosphorous compounds could produce polyphosphoric acid with strong dehydration ability that can rapidly make the oxygen-containing organic compound dehydration and carbonization, or even change the approach of thermal decomposition of the fuel. ${ }^{3}$ 9,10-dihydro-9-oxa-10-

Fujian Provincial Key Laboratory of Fire Retardant Materials, College of Materials, Xiamen University, Xiamen Fujian, P. R. China 361005. E-mail: brzeng@xmu.edu. cn; Fax: +86-0592-2183937; Tel: +86-18205926801

$\dagger$ Electronic supplementary information (ESI) available. See DOI: $10.1039 / \mathrm{c} 7 \mathrm{ra02529g}$ phosphaphenanthrene-10-oxide (DOPO) is a typical phosphorous-containing flame retardant which has high thermal stability, good oxidation and can significantly reduce the flammability and increase the char residue of polymeric materials. $^{4}$

Polyhedral oligomeric silsesquioxane (POSS) is a kind of inorganic-organic hybrid material with a nanosized, cageshaped and three-dimensional structure. The typical POSS molecule have general formula of $\mathrm{RSiO}_{1.5}$ where $\mathrm{R}$ is hydrogen or an organic group, such as alkyl, aryl or any of their derivatives. ${ }^{5}$ POSS have received wide attention and research due to their environmental neutrality, good heat resistance and excellent thermoxidative stability. Incorporation of the POSS into polymers can significantly improve their thermal properties and oxidation resistance as well as flame retardancy. ${ }^{6-8}$

It is worth noting that additives containing both silicon and phosphorus can achieve a synergistic flame-retardant effect, thus increasing the flame retardancy more effectively. ${ }^{9-12}$ For instance, Yang et al. ${ }^{\mathbf{1 3 , 1 4}}$ synthesized a flame-retardant (DOPOPOSS) as a good halogen-free flame retardant for polymers. Song et $a .^{15}$ and Hsiue et al. ${ }^{16}$ reported a novel organic/ inorganic epoxy hybrids containing phosphorus and silica that can promote the formation of a protective char, protecting the underlying materials from further burning. In our previous work, ${ }^{17}$ a novel DOPO-containing POSS with a high yield was synthesized via simple synthetic routes, and it could easily to self-assemble in EP and produce a good flame retardancy. However, the synergistic flame-retardant effect with metal has seldom been discussed till now. 
The metal can be introduced into polymer by covalent bond via certain reaction or dispersion as inorganic clusters. ${ }^{18}$ It is well known that the Lewis acidic metals can catalyze oxidative dehydrogenation reaction in gas phase.$^{19}$ In fact, the presence of the metal in the polymer will form more thermally stable phase, even promote partial matrix cross-linking, hence lead to the increase of thermal stability. ${ }^{20}$ Antonov et $a .^{21}$ reported that finely dispersed metals at low concentration ( $\leq 1 \mathrm{wt} \%$ ) strongly enhanced char yield.

In our work, we synthesized the compound POSS-bisDOPO containing two unit of DOPO and one unit of POSS, and used it as additives to construct a multi-element additive system containing phosphorus and silicon as well as titanium elements. As metal source, TBT is a kind of organic titanate and can react with hydroxyl group of epoxy, which could achieve very good dispersion in polymer matrix and have better catalytic effect. We used some characterization techniques, including TGA, LOI, UL-94, SEM, DMA and three-point bending test, to investigate the thermal degradation property and to discuss the flame retardancy mechanism of the prepared EP composites.

\section{Experimental}

\subsection{Materials}

Aminopropylisobutyl POSS (Product no. AM0265) was purchased from Hybrid Plastics Company. 9,10-Dihydroxa-10phosphaphenanthrene-10-oxide (DOPO) and tetrabutyl titanate (TBT) was purchased from Shanghai Eutec Chemical Co. Ltd., China. Diglycidyl ether of bisphenol A (DGEBA, trade name E51), a kind of liquid epoxy resin, was supplied by Jinan Qingtian Chemical Co. Ltd., China. 4,4'-Diaminodiphenylmethane (DDM) and polyoxymethylene (POM) were purchased from Sinopharm Chemical Reagent Co. Ltd., China. The reagents used were of analytical grade unless otherwise noted.

\subsection{Synthesis of POSS-bisDOPO}

POSS-bisDOPO was synthesized by aminopropylisobutyl POSS, DOPO and POM according to our previously reported procedure. ${ }^{17} \mathrm{NH}_{2}$-POSS (20 mmol, $17.48 \mathrm{~g}$ ), DOPO (50 mmol, $10.80 \mathrm{~g}$ ), POM (50 mmol, $1.50 \mathrm{~g}$ ) and chloroform $(120 \mathrm{~mL})$ were added into a $250 \mathrm{~mL}$ round-bottom glass flask equipped with a condenser and mechanical stirrer. The reaction mixture was stirred vigorously and heated to $61{ }^{\circ} \mathrm{C}$. The reaction was continued at $61{ }^{\circ} \mathrm{C}$ around for $24 \mathrm{~h}$ and then the solvent was concentrated to $20 \mathrm{~mL}$ by rotary evaporators. Afterward, the concentrated solvent slowly dropped into $400 \mathrm{~mL}$ cold methanol and then placed in the refrigerator for $10 \mathrm{~h}$. A lot of white precipitate was formed and filtered the solution through a suction filter device. Subsequently, the filter cake was dried under vacuum at $60^{\circ} \mathrm{C}$ for $24 \mathrm{~h}$ to get the target product (POSS-bisDOPO, yield, $25.3 \mathrm{~g}, 85 \%$ ) as a white powder. The synthesis route and characterization data were shown in the ESI (see Fig. S1†).

\subsection{Preparation of cured epoxy resin and its composites}

The cured epoxy resins were obtained using a thermal curing process. At first, TBT was dispersed in DGEBA by magnetic stirring at $105{ }^{\circ} \mathrm{C}$ for $1 \mathrm{~h}$ till dissolving completely. During the reaction process, the vacuum pump was used to remove small molecules formed in the reaction. And then, the POSS-bisDOPO was added into DGEBA at $105^{\circ} \mathrm{C}$ for $0.5 \mathrm{~h}$. After cooled to $85^{\circ} \mathrm{C}$, stoichiometric amounts of DDM was added. The mixture was poured into an aluminium mould once the mixture became homogeneous. Meanwhile, a group of pure epoxy resin was prepared as control group. All the composition of the samples were listed in Table 1 . All samples were cured at $120^{\circ} \mathrm{C}$ for $4 \mathrm{~h}$, followed by at $140{ }^{\circ} \mathrm{C}$ for $2 \mathrm{~h}$. They were then further post-cured at $180{ }^{\circ} \mathrm{C}$ for $2 \mathrm{~h}$ to ensure the complete curing reaction.

\subsection{Characterization}

The surface morphology of structure of the fracture and the char residues from the LOI tests were examined with TM3000 tabletop microscope, using an acceleration voltage of $15 \mathrm{kV}$. Thermogravimetric analysis (TGA) was performed with a Netzsch STA 409EP by heating from room temperature to $800{ }^{\circ} \mathrm{C}$ at a heating rate of $10{ }^{\circ} \mathrm{C} \mathrm{min}{ }^{-1}$ under air and nitrogen atmospheres. Differential scanning colourimetry (DSC) was conducted with a Netzsch STA $449 \mathrm{C}$ by heating from room temperature to $250{ }^{\circ} \mathrm{C}$ at a heating rate of $10{ }^{\circ} \mathrm{C} \min ^{-1}$ under nitrogen atmosphere. The mass of each sample was approximately 4-10 mg. The sample transmittance was evaluated by UV2550 Spectrophotometer. The limited oxygen index (LOI) was determined with Fire Testing Technology Oxygen Index, and the specimen dimensions were $100 \mathrm{~mm} \times 6 \mathrm{~mm} \times 4 \mathrm{~mm}$. The

Table 1 Component and $T_{\mathrm{g}}$ of the cured EP composites

\begin{tabular}{|c|c|c|c|c|c|c|c|}
\hline Sample & DGEBA (g) & TBT $(\mathrm{g})$ & POSS-bisDOPO (g) & $\mathrm{Ti}$ & $\mathrm{P}$ & $\mathrm{Si}$ & $\frac{T_{\mathrm{g}}\left({ }^{\circ} \mathrm{C}\right)}{\mathrm{DSC}}$ \\
\hline POSS-bisDOPO & - & - & - & - & 4.66 & 16.84 & - \\
\hline EP0-0 & 100 & 0 & 0 & 0 & 0 & 0 & 155.1 \\
\hline EP1-9 & 100 & 1 & 9 & 0.14 & 0.42 & 1.52 & 150.6 \\
\hline EP10-0 & 100 & 10 & 0 & 1.40 & 0 & 0 & 179.6 \\
\hline EP0-10 & 100 & 0 & 10 & 0 & 0.46 & 1.68 & 145.9 \\
\hline
\end{tabular}


percentage of $\mathrm{O}_{2}$ in the $\mathrm{O}_{2} / \mathrm{N}_{2}$ mixture was taken as the LOI value, which was just sufficient to sustain the flame. Vertical burning tests were performed according to UL94 standard by Fire Testing Technology UL94, and the dimensions of each sample were $125 \mathrm{~mm} \times 12.5 \mathrm{~mm} \times 4 \mathrm{~mm}$. The reported results are the average of five measurements. Microscale combustion calorimeter (MCC) tests were conducted with a Fire Testing Technology calorimeter of FAA model. During testing, about $5 \mathrm{mg}$ of the sample was heated to $700{ }^{\circ} \mathrm{C}$ at a heating rate of $1^{\circ} \mathrm{C}$ $\mathrm{s}^{-1}$ in a stream of nitrogen flowing at $80 \mathrm{~mL} \mathrm{~min}^{-1}$. The thermal degradation products of the sample in nitrogen were mixed with a $20 \mathrm{~mL} \mathrm{~min}{ }^{-1}$ stream of oxygen prior to entering combustion furnace. Dynamic mechanical analysis (DMA) was carried out with a Netzsch DMA 242 E. The dimensions of the specimens were $60 \mathrm{~mm} \times 10 \mathrm{~mm} \times 3.2 \mathrm{~mm}$. The measuring frequency was $1 \mathrm{~Hz}$. The temperature was varied in the range of $30-220{ }^{\circ} \mathrm{C}$ with a heating rate of $1{ }^{\circ} \mathrm{C} \mathrm{min}^{-1}$. Three-point bending experiments were conducted on an electronic universal testing machine (AGS-X, Shimadzu, Japan). The crosshead speed was $1 \mathrm{~mm} \mathrm{~min}^{-1}$, and the typical dimension of the sample beams were $120 \mathrm{~mm} \times 10 \mathrm{~mm} \times 5 \mathrm{~mm}$.

\section{Results and discussion}

\subsection{Glass transition behavior}

A series of cured epoxy resins with different composition ratio and the calculated flame retardant element contents of the prepared EP composites as well as the corresponding glass transition temperature $T_{\mathrm{g}}$ from DSC were all given in Table 1. The DSC curves of the cured neat epoxy and its composites were shown in Fig. S2. $\dagger$ It can be seen that compared to neat EP, the addition of single component of TBT made the $T_{\mathrm{g}}$ of EP100 increase from $155.1{ }^{\circ} \mathrm{C}$ to $179.6{ }^{\circ} \mathrm{C}$. It was explained that TBT could react with hydroxyl groups of epoxy and serve as the crosslinking agent for epoxy. The increasing of crosslinking density of EP restrained the motion of segmers, consequently increased $T_{\mathrm{g}}$. However, the addition of single component of POSS-bisDOPO made the $T_{\mathrm{g}}$ of EP0-10 decrease from $155.1^{\circ} \mathrm{C}$ to $145.9^{\circ} \mathrm{C}$. The reason was that the dispersion of POSS-bisDOPO in EP lead to the increasing of the free volume of the system, which enlarged the motion space and improved the motion ability of segmers, thus resulting in a decline of $T_{\mathrm{g}}$. When the added ratio of TBT to POSS-bisDOPO was adjusted, the cured EP composites with different $T_{\mathrm{g}}$ could be obtained. It showed that the sample EP5-5 had a $T_{\mathrm{g}}$ of $161.3{ }^{\circ} \mathrm{C}$, which was higher than that of EP1-9 and EP3-7. Compared to EP0-0, the $T_{\mathrm{g}}$ of EP5-5 was obviously enhanced.

\subsection{Morphology characterization}

Because the synthesized POSS-bisDOPO is a kind of halogenfree flame retardant without reactive group on the molecular structure, we investigated the compatibility between the additive and epoxy resin by UV-vis and SEM. Fig. 1 displayed the photographs and transmittance curves of the cured EP composites. It can be seen from these photographs that, neat EP0-0 was clear and transparent, while the composite EP0-10 was a little blurry. Apparently, the addition of POSS-bisDOPO made the transmittance of composite EP get worse in the visible light range of $390-780 \mathrm{~nm}$, which was verified in the transmittance curves. For example, under the wavelength of $600 \mathrm{~nm}$, the transmittance of EP0-0, EP5-5, EP3-7, EP1-9 and EP0-10 were about $90 \%, 56 \%, 52 \%, 48 \%$ and $36 \%$, respectively. This implied that the transmittance of the cured samples declined as the loaded amount of POSS-bisDOPO increased. Although the physical dispersing of POSS-bisDOPO in DGEBA could lead to the decrease of transmittance of cured samples, the EP composites did not show an obvious phase separation because POSS-bisDOPO can self-assembly in DGEBA to form small particles with nanosize. ${ }^{17}$ When it turned to TBT, we found that the addition of TBT played a weaker effect on the transparency of composite EP in the visible light range. For instance, under the wavelength of $600 \mathrm{~nm}$, the transmittance of EP10-0 was about $84 \%$, which only had a decreasing of $6 \%$ comparing to pure EP. This may be attributed to the reactivity of TBT with certain groups such as hydroxyl on epoxy monomers. These results indicated that the high affinity of TBT for DGEBA kept the cured EP composites with a good transmittance.

SEM characterization was used to further understand the micro-morphologies of the fracture surfaces of cured epoxy resin and its composites. Fig. 2 showed the SEM images of the fracture surfaces of the cured EP composites. It can be observed from Fig. 2A that neat epoxy resin (EP0-0) had a smooth fracture
(A)

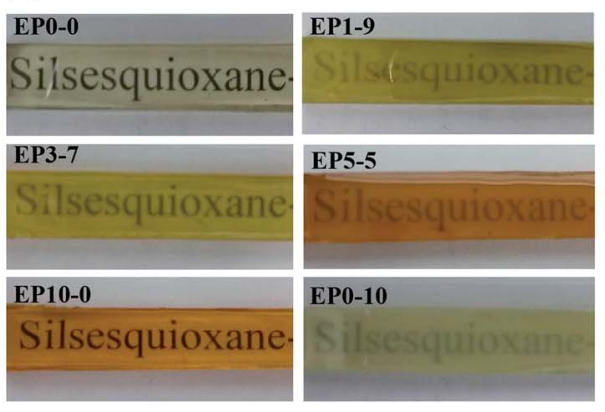

(B)

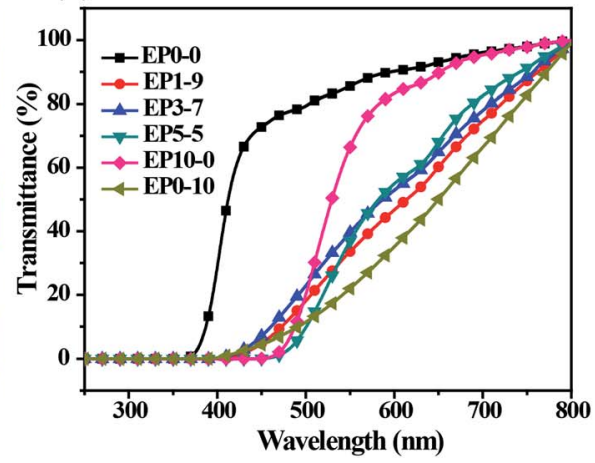

Fig. 1 (A) Photographs and (B) transmittance curves of the cured EP composites. 

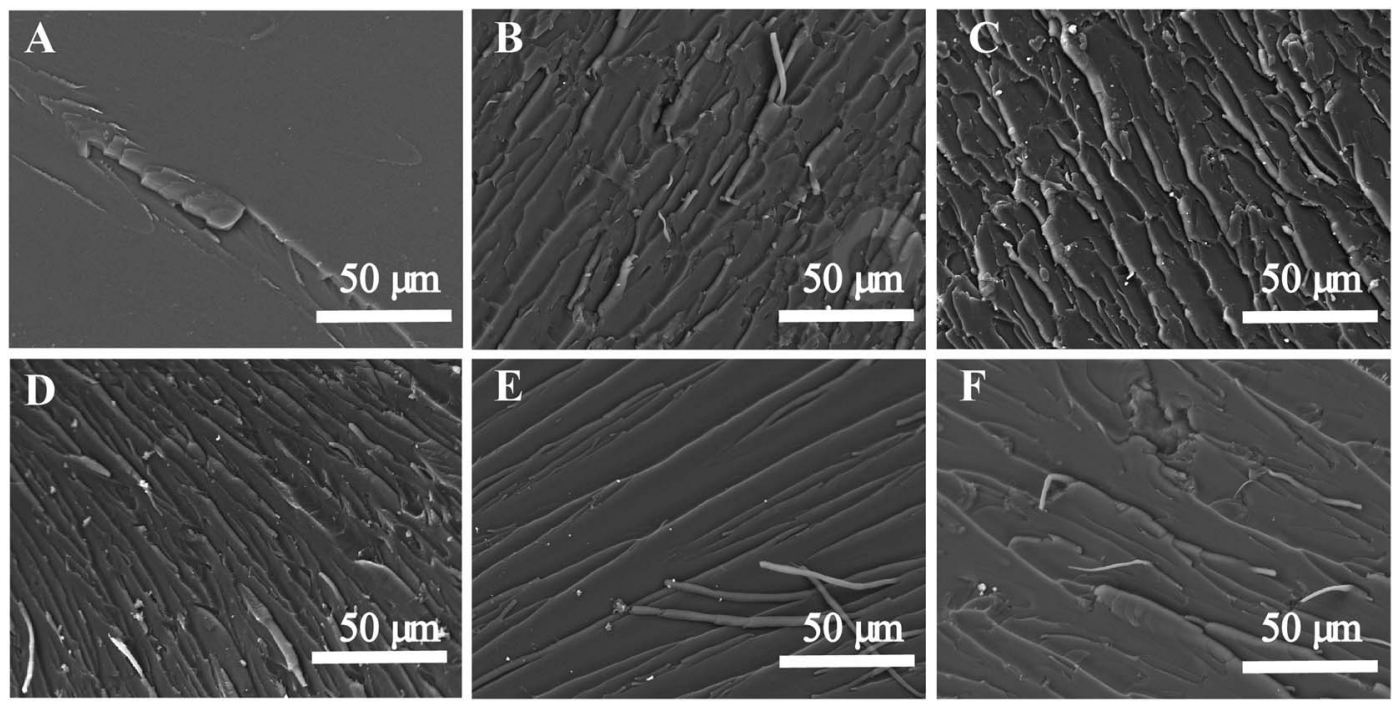

Fig. 2 SEM images of the fracture surfaces of the cured EP composites. (A) EP0-0, (B) EP1-9, (C) EP3-7, (D) EP5-5, (E) EP10-0, (F) EP0-10.

surface and showed a typical brittle fracture. However, when 10 wt $\%$ TBT and $10 \mathrm{wt} \%$ POSS-bisDOPO was incorporated respectively, both the prepared EP10-0 and EP0-10 occurred a completely different fracture, which was shown in Fig. 2E and F respectively. From the surface morphology, we observed that the distribution of fracture stripe increased and the fracture area turned relatively larger, which indicated the transition of the fracture behavior from brittle fracture to ductile fracture after the incorporation of additives. On one hand, because POSS-bisDOPO physically dispersed in DGEBA, it could improve the motion ability of segmers as plasticizers did, and then lead to a toughening effect on the cured EP composites. On the other hand, because the addition of TBT enhanced the density of cross linking of cured matrix, it would increased the energy barrier for the fracture to overcome. When POSS-bisDOPO and TBT were added together into EP, such as EP1-9, EP3-7 and EP55 , it could be seen that the distribution of fracture stripe further increased with an uneven surface, which indicated a better fracture toughness.

\subsection{Mechanical property}

The mechanical properties of cured epoxy resin and its composites were evaluated by dynamic mechanical analysis under double cantilever model. The curves of storage modulus and loss factor $\tan \delta$ were shown in Fig. 3, and the data are also given in the ESI (see Table S1 $\dagger$ ). It showed that the storage modulus of the neat epoxy (EP0-0), EP1-9, EP3-7, EP5-5, EP10-0, and $\mathrm{EP} 0-10$ at $40{ }^{\circ} \mathrm{C}$ were $1245,1350,1235,1247,1244$ and $1241 \mathrm{MPa}$, respectively. This implied that at the glass state, the cured EP composites with the incorporation of POSS-bisDOPO and TBT almost kept the same dynamic mechanical properties with a small variation. When the temperature rose to $190{ }^{\circ} \mathrm{C}$, the cured EP composites were in a rubber state. The storage modulus of the EP0-0 was $10.1 \mathrm{MPa}$, while that of EP100 and EP0-10 increased to $19.6 \mathrm{MPa}$ and $11.1 \mathrm{MPa}$, respectively. In addition, all the storage modulus of EP1-9, EP3-7 and EP5-5 were enhanced to a higher level compared to neat EP. It was explained that the introduction of TBT increased the crosslinking density of EP system, and then enhanced the storage modulus of cured EP composites under the rubber state.

In order to further investigate the mechanical property of the epoxy resin, three-point bending tests were used to measure the elastic modulus of the cured EP composites. The experimental results showed that the elastic modulus of the EP0-0 was $2559 \mathrm{MPa}$, while those of the composited EP1-9, EP3-7, EP5-5, EP10-0 and EP0-10 were 2620, 2588, 2563, 2595, and $2631 \mathrm{MPa}$, respectively. The increasing of elastic modulus implied that the mechanical property of the epoxy resin composites have no decline by the incorporation of POSSbisDOPO and TBT. The result was consistent with the above dynamic mechanical analysis.

\subsection{Thermal stability}

TGA is an effective method for characterizing the thermo oxidation resistance of materials. Fig. 4 provided the TGA curves

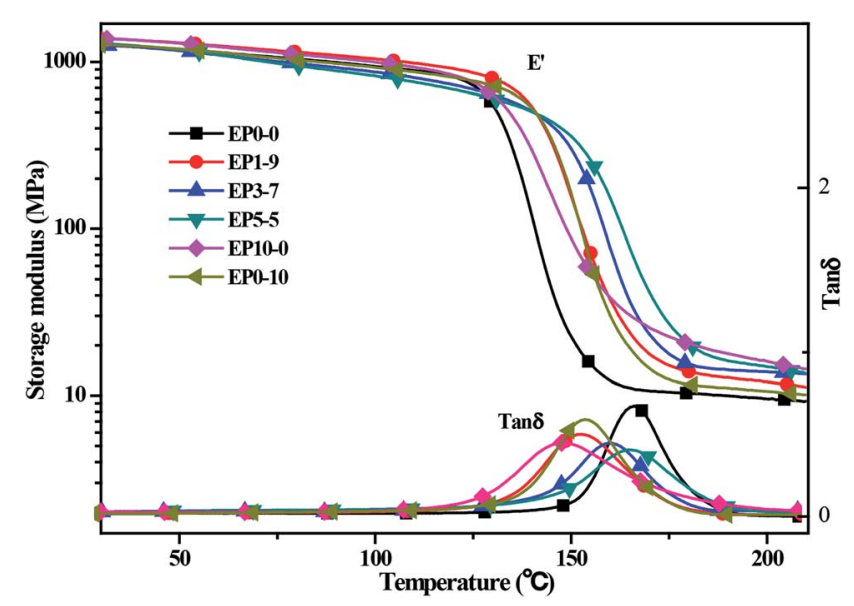

Fig. 3 DMA curves of the cured EP composites. 

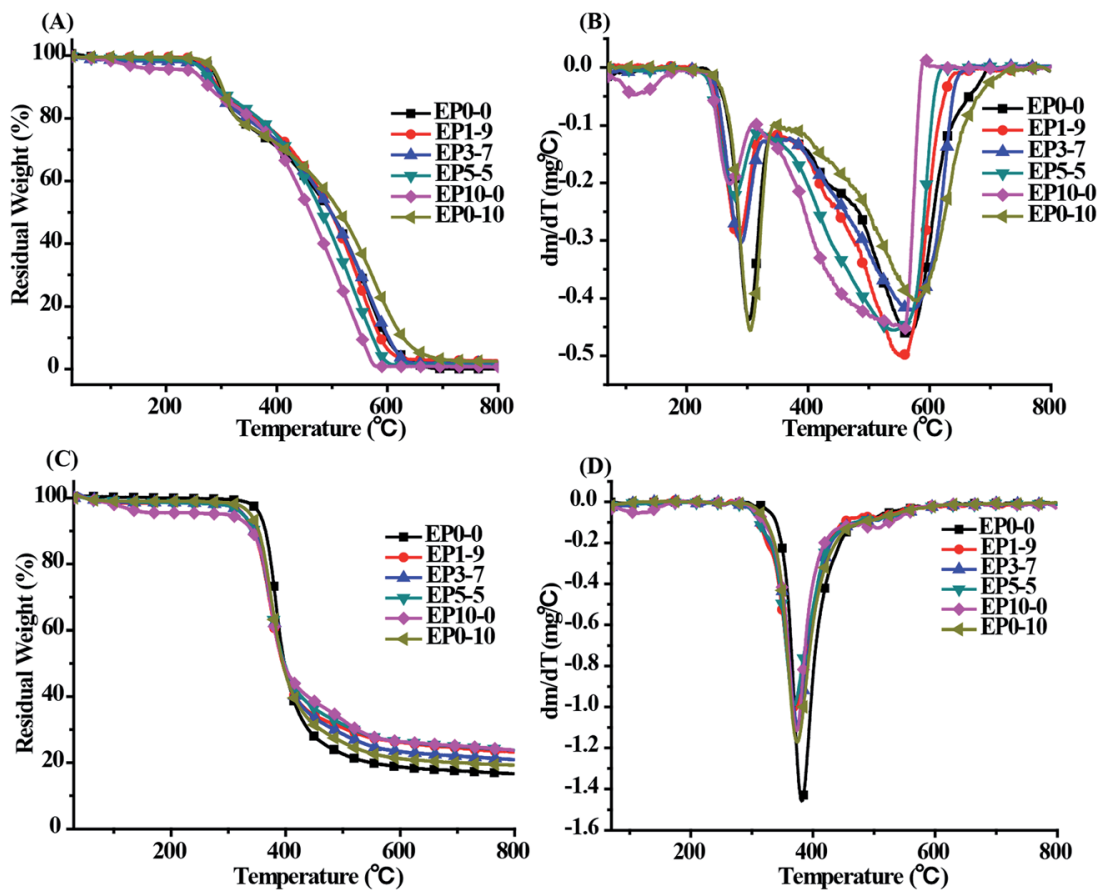

Fig. 4 TGA (left) and DTG (right) curves of the cured EP composites. (A and B) Under air atmosphere, (C and D) under nitrogen atmosphere, $10{ }^{\circ} \mathrm{C} \mathrm{min}^{-1}$.

of the cured neat epoxy and its composites under both air and nitrogen atmospheres. The relevant thermal decomposition data, including $T_{\mathrm{d}}$ which was defined as the temperature at 5 wt $\%$ weight loss, and $T_{\max }$ which was defined as the temperature at maximum weight loss rate, and the char residues at $800{ }^{\circ} \mathrm{C}$, are given in Table 2 .

As shown in Fig. 4, under the nitrogen atmospheres, the $T_{\mathrm{d}}$ and $T_{\max }$ of EP0-0 were around $354.5^{\circ} \mathrm{C}$ and $380.9{ }^{\circ} \mathrm{C}$, respectively, and with a maximum weight loss rate being $1.45 \mathrm{wt} \%$ $\min ^{-1}$. However, the $T_{\mathrm{d}}$ and $T_{\max }$ of the composites (EP1-9EP10-0) are in the range of $290-340{ }^{\circ} \mathrm{C}$ and $360-375{ }^{\circ} \mathrm{C}$, respectively, which was lower than that of neat epoxy (EP0-0). The result could be ascribed to the relatively low temperature of the degradation of the phosphorus and titanate group, which mainly caused by the weak $\mathrm{P}-\mathrm{O}-\mathrm{C}$ and $\mathrm{Ti}-\mathrm{O}-\mathrm{C}$ bond decomposition in POSS-bisDOPO and TBT. This phenomenon has also been observed in other literatures. ${ }^{22,23}$ Besides, the maximum weight loss rate of EP1-9, EP3-7, EP5-5, EP0-10 and EP10-0 were

Table 2 Thermal decomposition data of the cured EP composites

\begin{tabular}{|c|c|c|c|c|c|c|c|}
\hline \multirow[b]{2}{*}{ Sample } & \multicolumn{4}{|l|}{ Air } & \multicolumn{3}{|c|}{ Nitrogen } \\
\hline & $T_{\mathrm{d}}$ & $T_{\max 1}$ & $T_{\max 2}$ & Char & $T_{\mathrm{d}}$ & $T_{\max 1}$ & Char \\
\hline ТВТ & - & - & - & 23.53 & - & - & - \\
\hline POSS-bisDOPO & 332.9 & 433.9 & 501.9 & 52.60 & 302.9 & 369.9 & 4.27 \\
\hline EP0-0 & 284.5 & 302.2 & 566.3 & 0.05 & 354.5 & 380.9 & 16.67 \\
\hline EP1-9 & 275.1 & 286.1 & 553.5 & 2.63 & 326.5 & 370.7 & 23.19 \\
\hline EP3-7 & 271.0 & 289.5 & 570.2 & 1.97 & 325.9 & 374.3 & 20.91 \\
\hline EP5-5 & 267.5 & 276.9 & 540.2 & 1.27 & 325.1 & 368.9 & 23.75 \\
\hline EP10-0 & 246.9 & 271.8 & 556.6 & 0.76 & 297.3 & 373.6 & 23.75 \\
\hline EP0-10 & 287.5 & 303.4 & 577.0 & 2.45 & 338.5 & 373.9 & 19.25 \\
\hline
\end{tabular}

about $0.99,1.01,1.08,1.11$ and $1.17 \mathrm{wt}^{2} \mathrm{~min}^{-1}$, respectively. Notably, the maximum weight loss rate of the cured EP composites were slower than that of neat epoxy. Furthermore, the cured EP composites with both POSS-bisDOPO and TBT had a slower maximum weight loss rate relative to the composites alone added POSS-bisDOPO or TBT. This might be explained that phosphorus-containing and silicon-containing flame retardants can reduce the degradation rate of the epoxy resins. ${ }^{9,15,16}$ However, when the system included all the three elements of phosphorus and silicon as well as titanium, the degradation rate further decreased. This phenomenon may be due to the interaction of phosphorous, silicon and titanium, resulting in a synergistic effect during thermal degradation.

Under the air atmospheres, the first decomposition process of all samples occurred at around $300{ }^{\circ} \mathrm{C}$, implying that these samples adopt the same thermal decomposition pathway. This is probably induced by the dehydrogenation and aromatization of alkyl groups at this temperature. ${ }^{24} \mathrm{Next}$, a second degradation stage appeared at $500-700{ }^{\circ} \mathrm{C}$, which is considered to be the thermo-oxidative degradation of the char. Similarly, the degradation rate decreased by adding POSS-bisDOPO and TBT. As shown in Table 2, all of the values of the char residues of composites in the experiment were higher than the theoretical values, which might attribute to the synergistic effect of phosphorous, silicon and titanium. It has been reported that phosphorous can accelerate the formation of char, silicon could form a ceramic-like layer to keep the char from undergoing thermal degradation. ${ }^{25}$ Moreover, the catalytic effect of metals on polymer showed that finely dispersed metals at low concentration strongly enhanced char yield by catalyzing dehydrogenation during polymer thermal degradation. ${ }^{21}$ 
Table 3 LOI values and UL-94 data of the cured EP composites

\begin{tabular}{llllll}
\hline & & & & \\
\cline { 3 - 5 } & & & & \\
Sumple & LOI $(\%)$ & gurning & & \\
grade & $t_{1}(\mathrm{~s})$ & $t_{2}(\mathrm{~s})$ & Dripping \\
\hline EP0-0 & 26.2 & NR & $>30$ & - & Yes \\
EP1-9 & 30.5 & V-0 & 2 & 3 & No \\
EP3-7 & 31.2 & V-0 & 3 & 6 & No \\
EP5-5 & 31.0 & V-0 & 2 & 5 & No \\
EP10-0 & 28.0 & V-1 & 18 & 2 & No \\
EP0-10 & 30.3 & V-1 & 27 & 3 & No \\
& & & & &
\end{tabular}

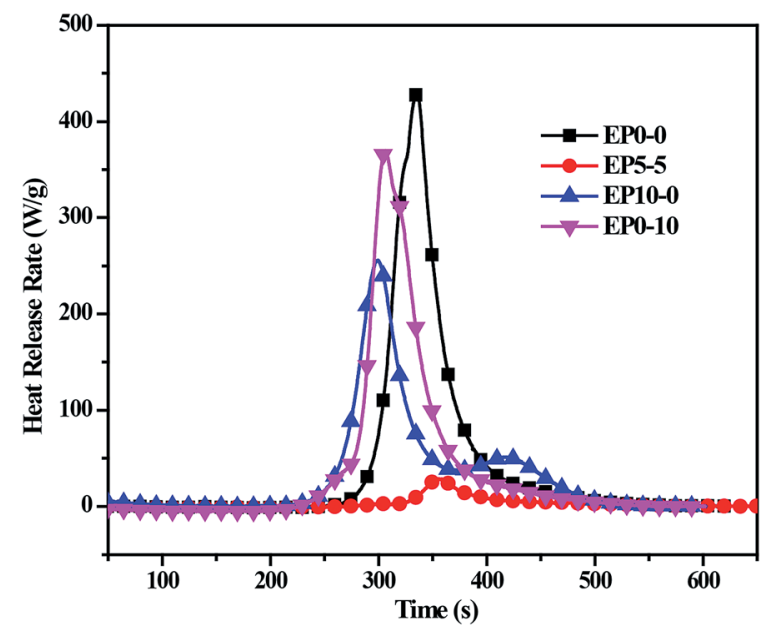

Fig. 5 HRR curves of the cured EP composites.

Based on the above discussion, it was considered that a synergistic effect of phosphorous, silicon and titanium happened in the system of cured EP composites during the thermal degradation. The phosphorous and silicon is helpful to form a stable, continuous and compact condensed phase which can prevent the diffusion of the heat and the oxygen to the inner matrix. Meanwhile, the titanium could be activated under the heat and then play a role of catalyst which can accelerate the formation of char. The synergistic effect increased the char residues and decreased the degradation rate of epoxy resins by forming a more compact physical protective layer.

\subsection{Flame retardancy}

The effect of POSS-bisDOPO and TBT on the LOI values and UL94 ratings of the cured EP composites are presented in Table 3. We could find that the LOI values of the cured EP composites increased from $26.2 \%$ to $31.2 \%$. The LOI values of EP0- 10 $(30.3 \%)$ is higher than that of EP10-0 (28.0\%), revealing that POSS-bisDOPO had a better flame retardance than TBT. Compared with the composite just added POSS-bisDOPO (EP010), the LOI values of other composites simultaneously possessing POSS-bisDOPO and TBT (EP1-9, EP3-7, EP5-5) had no obvious improvement, which demonstrated that the TBT played a weak role in affecting the LOI value of the epoxy resin. However, the UL-94 test indicated that the synergetic effect of phosphorous, silicon and titanium may occur in the cured EP composites during combustion. All of the cured EP composites had no dripping and could self-extinguishing. When only POSSbisDOPO or TBT was incorporated, the UL-94 rating was V-1. However, an interesting result can be observed that the UL-94 ratings of composites raised to V-0 when POSS-bisDOPO and TBT were added together. In summary, the mixture of POSSbisDOPO and TBT had a remarkable positive effect in flame retardancy during combustion.

The heat release rate (HRR) curves of the cured EP composites were displayed in Fig. 5 , the peak heat release rate (PHHR) and the total heat release (THR) were given in Table S2. $\dagger$ It can be seen that the PHHR and the THR of EP0-0 are $430.2 \mathrm{~W} \mathrm{~g}^{-1}$ and $22.7 \mathrm{~kJ} \mathrm{~g}^{-1}$, respectively. When only POSSbisDOPO or TBT was incorporated (EP0-10, EP10-0), the PHHR decreased to 369.2 and $255.6 \mathrm{~W} \mathrm{~g}^{-1}$, respectively. It is worth noting that the PHHR of the composites with both POSS-
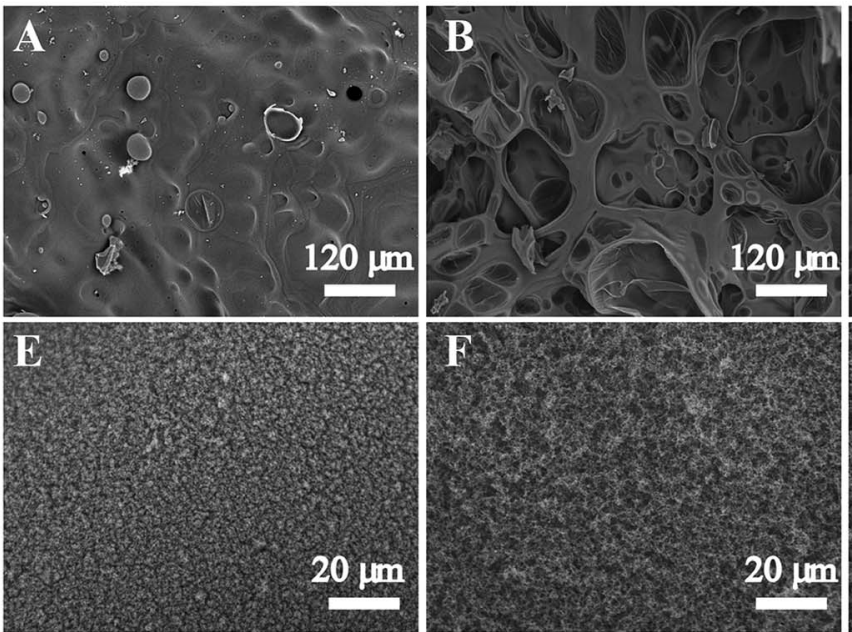
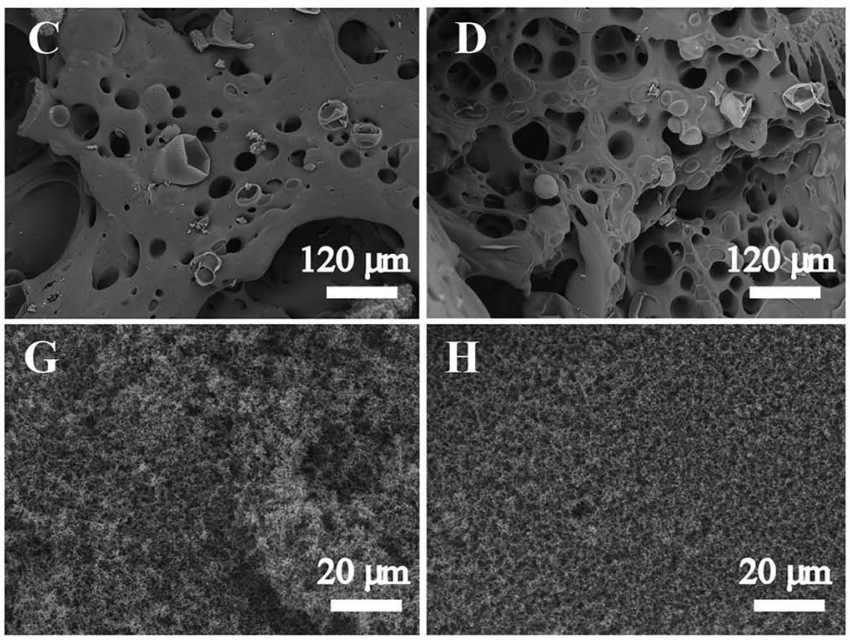

Fig. 6 SEM images of interior char residue of the cured EP composites (top) and SEM images of exterior char residue of the cured EP composites (bottom). (A and E) EPO-O, (B and F) EP5-5, (C and G) EP10-0, (D and H) EP0-10. 
bisDOPO and TBT (EP5-5) sharply decreased to $28.4 \mathrm{~W} \mathrm{~g}^{-1}$. Furthermore, the THR of EP5-5 also significantly decreased relative to EP0-10 and EP10-0, the THR of EP5-5, EP0-10 and EP10-0 are 1.7, 20.1 and $17.9 \mathrm{~kJ} \mathrm{~g}^{-1}$, respectively. The results indicated that the incorporation of POSS-bisDOPO and TBT decreased the HHR and the THR of the cured EP composites, and the coexistence of phosphorous, silicon and titanium played a good synergistic flame retardant effect.

\subsection{Morphology characterization of char residue}

The microstructure of char residues of the cured neat epoxy and its composites were characterized by SEM and shown in Fig. 6. It can be seen that the char of neat epoxy resin presented a smooth inner surface (Fig. 6A) and a loose outer layer with grain-like structure (Fig. 6E), which resulted from rapid volatilization of the condensed phase to the outer. The drawback of this kind of residue is that the heat and oxygen could easily penetrate through this char and enter into the inner, consequently increased the combustion intensity. However, after adding POSS-bisDOPO and TBT, the char residue of the cured EP composites displayed an alveolate inner layer with some broken bubbles on the surface (Fig. 6B-D). Moreover, a continual and compact exterior char layer was formed (Fig. 6F-H), which was helpful to prevent the volatile gas from thermal degradation from spreading. In summary, the tougher and compact char can hinder the heat and oxygen, and ultimately weaken the combustion intensity.

\section{Conclusions}

Together with tetrabutyl titanate, the compound POSS-bisDOPO consisting of two unit of DOPO and one unit of POSS was used as flame retardant additives to improve the flame retardancy of cured epoxy resin. The cured EP composites with different ratio of TBT to POSS-bisDOPO were prepared and characterized by DSC, SEM, DMA, TGA, LOI, UL-94, and three-point bending test etc. Compared to pure EP, the char yield of the prepared EP composite was increased from $16.67 \%$ to $23.75 \%$. The LOI value and the UL-94 ratings of the cured EP composites with a loading of POSS-bisDOPO being $5 \%$ were also raised up to $31.0 \%$ and V0 grade, respectively. Meanwhile, the PHRR and THR value reduced to $28.4 \mathrm{~W} \mathrm{~g}^{-1}$ and $1.7 \mathrm{~kJ} \mathrm{~g}^{-1}$ respectively. The SEM results indicated that the residual char of the prepared EP composites had an alveolate inner layer and continual compact outer layer, which was conducive to form effectively physical barrier to prevent the penetrating of the heat and oxygen. The DMA and three-point bending test results showed that the mechanical property of the prepared EP composites had no decline. In this work, the combination of POSS-bisDOPO and TBT can not only obviously improve the flame retardancy of the cured EP composites, but also remain their good mechanical property. During combustion, the synergistic effect of phosphorous, silicon and titanium accelerated the formation of condensed phase and provided continual and compact char layer that prevented further combustion of polymer.

\section{Acknowledgements}

This work was supported by the National Natural Science Foundation of China (51573150, U1205113), Scientific and Technological Innovation Platform of Fujian Province (2014H2006), National Science Technology Program (2014BAF08B03), and Key Project of Fujian Department Science and Technology (2013HZ0005-1).

\section{References}

1 J. Wan, C. Li, Z. Y. Bu, C. J. Xu, B. G. Li and H. Fan, Chem. Eng. J., 2012, 188, 160-172.

2 R. Liu and X. Wang, Polym. Degrad. Stab., 2009, 94, 617-624.

3 J. Liu, J. Y. Tang, X. D. Wang and D. Z. Wu, RSC Adv., 2012, 2, 5789-5799.

4 K. A. Salmeia and S. Gaan, Polym. Degrad. Stab., 2015, 113, 119-134.

5 G. Li, L. Wang, H. Ni and C. U. Pittman, J. Inorg. Organomet. Polym., 2001, 11, 123-154.

6 S. W. Kuo and F. C. Chang, Prog. Polym. Sci., 2011, 36, 1649-1696.

7 D. Gnanasekaran, K. Madhavan and B. S. R. Reddy, J. Sci. Ind. Res., 2009, 68, 437-464.

8 Y. Qian, P. Wei, X. Zhao, P. Jiang and H. Yu, Fire Mater., 2013, 37, 1-16.

9 W. C. Zhang, X. M. Li and R. J. Yang, Polym. Degrad. Stab., 2011, 96, 2167-2173.

10 P. J. Chao, Y. J. Li, X. Y. Gu, D. D. Han, X. Q. Jia, M. Q. Wang, T. F. Zhou and T. Wang, Polym. Chem., 2015, 6, 2977-2985.

11 S. Q. Song, J. J. Ma, K. Cao, G. J. Chang, Y. W. Huang and J. X. Yang, Polym. Degrad. Stab., 2014, 99, 43-52.

12 C. L. Chiang, R. C. Chang and Y. C. Chiu, Thermochim. Acta, 2007, 453, 97-104.

13 W. C. Zhang, X. M. Li and R. J. Yang, Polym. Degrad. Stab., 2012, 97, 1314-1324.

14 W. C. Zhang, X. M. Li and R. J. Yang, Polym. Degrad. Stab., 2014, 99, 118-126.

15 X. D. Qian, L. Song, Y. Hu and R. K. K. Yuen, Thermochim. Acta, 2013, 552, 87-97.

16 G. H. Hsiue, Y. L. Liu and J. Tsiao, J. Appl. Polym. Sci., 2000, 78, 1-7.

17 C. Liu, T. Chen, C. H. Yuan, C. F. Song, Y. Chang, G. R. Chen, Y. T. Xu and L. Z. Dai, J. Mater. Chem. A, 2016, 4, 3462-3470. 18 G. Kickelbick, Prog. Polym. Sci., 2003, 28, 83-114.

19 M. A. Banares, Catal. Today, 1999, 51, 319-348.

20 A. Fina, H. C. L. Abbenhuis, D. Tabuani, A. Frache and G. Camino, Polym. Degrad. Stab., 2006, 91, 1064-1070.

21 A. Antonov, M. Yablokova, L. Costa, A. Balabanovich, G. Levchik and S. Levchik, Mol. Cryst. Liq. Cryst. Sci. Technol., Sect. C, 2000, 353, 203-210.

22 X. Wang, Y. Hu, L. Song, W. Y. Xing, H. D. A. Lu, P. Lv and G. X. Jie, Polymer, 2010, 51, 2435-2445.

23 M. Selvaraj, K. Maruthan, S. Palraj and G. Venkatachari, Prog. Org. Coat., 2010, 67, 339-347.

24 K. Wu, L. Song, Y. Hu, H. D. Lu, B. K. Kandola and E. Kandare, Prog. Org. Coat., 2009, 65, 490-497.

25 X. Wang, Y. A. Hu, L. Song, W. Y. Xing and H. D. Lu, J. Polym. Sci., Part B: Polym. Phys., 2010, 48, 693-705. 